\title{
High power ultrasound: An innovation in the food processing industry
}

\author{
Latika Yadav, Vibha Bhatnagar and V.D. Mudgal
}

\begin{abstract}
Power ultrasound is an emerging and promising technology for food processing industry. Pressing demands from consumers for quality food products with natural flavor and taste, free from additives and preservatives, has triggered need for the development of non-thermal process methods which offer maximum quality and safety of food products. Ultrasound is considered as one such nonthermal processing alternative, which can be used in many food processing operations. It travels through a medium like any sound wave, resulting in a series of compression and rarefaction. Due to their important features at ambient or lower temperatures, the non-thermal technologies are regarded as potential and powerful tools in food processing. High energy ultrasound $(20-500 \mathrm{kHz})$ is referred to as High power ultrasound (HPU). The physical, mechanical or chemical effects of high power ultrasound are capable of altering material properties. These effects are promising in food processing, preservation and safety. It has been realized that HPU has much to offer to the food industry such as drying, extraction, crystallization, defoaming, emulsification, inactivation of microorganism and enzymes, filtration, separation, etc of valuable compounds from vegetables and food products. In this paper a review of the most recent uses of power ultrasound in the food industry will be discussed.
\end{abstract}

Key Words : High power ultrasound, Innovation, Food processing industry

How to cite this article : Yadav, Latika, Bhatnagar, Vibha and Mudgal, V.D. (2014). High power ultrasound: An innovation in the food processing industry. Food Sci. Res. J., 5(2): 190-196.

Associate Authors' :

VIBHA BHATNAGAR, Department of Foods and Nutrition, College of Home Science, Maharana Pratap University of Agriculture and Technology, UDAIPUR (RAJASTHAN) INDIA

Email: vibhasuresh@gmail.com

V.D. MUDGAL, Department of Processing and Food Engineering, College of Technology and Engineering, Maharana Pratap University of Agriculture and Technology, UDAIPUR (RAJASTHAN) INDIA

Email: mudgalvd@gmail.com 